\title{
DOUBLE-MASKED THREE-PERIOD CROSSOVER INVESTIGATION OF TIMOLOL IN CONTROL OF RAISED INTRAOCULAR PRESSURE
}

\author{
S. H. CAMPBELL, M. HICKEY-DWYER and S. P. HARDING \\ Liverpool
}

\begin{abstract}
SUMMARY
Forty patients with an intraocular pressure (IOP) between 30 and $49 \mathrm{mmHg}$ in either eye (57 eyes) were recruited into a trial of timolol $0.25 \%$ versus timolol $0.5 \%$. Patients were randomised to receive the two concentrations of drug in the order ABA or BAB. The study period was 12 weeks for each patient, with changes in drug concentration at 4 and 8 weeks. IOPs were measured at the same time every 2 weeks. Mean IOP of both eyes was used, excluding any eyes with initial IOPs of less than $30 \mathrm{mmHg}$. There was no difference between the 2-week and 4-week pressure readings, which were therefore combined. There was no statistically significant difference in the initial IOPs of the two groups $(0.5 \%$, $33.45 \mathrm{mmHg} ; 0.25 \%, 32.63 \mathrm{mmHg}$ ) nor in the initial fall in IOP with either concentration $(0.5 \%, 12.03 \mathrm{mmHg}$; $0.25 \%, 11.31 \mathrm{mmHg}$ ). Furthermore, changing from one concentration to the other had no significant effect on IOP. Mean IOPs over the whole study period averaged $21.12 \mathrm{mmHg}$ for timolol $0.25 \%$ and $20.75 \mathrm{mmHg}$ for timolol $0.5 \%$. Again these differences were not statistically significant. The statistical power of the study was estimated to exceed $85 \%$. The authors suggest that there is no justification for use of the $0.5 \%$ strength, which is more expensive and has no advantages.
\end{abstract}

Timolol maleate is a non-selective, beta- 1 and beta- 2 adrenergic antagonist. It is available in the United Kingdom in two concentrations: $0.25 \%$ and $0.5 \%$. The $0.5 \%$ concentration currently accounts for two thirds of prescriptions* despite previous work suggesting that the higher concentration was no more effective at lowering intraocular pressure (IOP). ${ }^{1,2}$ In 1987 Novack $^{3}$ reported

From: St. Paul's Eye Unit, Royal Liverpool Hospital, Liverpool, UK Correspondence to: S. H. Campbell, Kent County Ophthalmic and Aural Hospital, Church Street, Maidstone, Kent, UK.

*Annual UK sales figures for timolol $(0.25 \%$ and $0.5 \%)$, hospital and retail combined: $0.25 \%=1,179,900$ units $=39 \% ; 0.5 \%=1,826,700$ units $=61 \%$. that both these studies ${ }^{1,2}$ suffered from low statistical power. The aim of this study, therefore, was to answer conclusively the question: 'Is there any difference in efficacy between the two available concentrations of timolol?' The study was designed to exceed the $80 \%$ statistical power level.

\section{PATIENTS AND METHODS}

This study followed the same recruitment criteria and methods as the study comparing metipranolol $0.1 \%, 0.3 \%$ and $0.6 \%$ which preceded it. ${ }^{4}$ Previously undiagnosed patients with an IOP of $30-49 \mathrm{mmHg}$ were eligible for inclusion in the study, as were those with known open angle glaucoma controlled on beta blockers alone, although no patients in this category were recruited. Patients with advanced disc cupping and field loss, concomitant ocular disease, or known contraindications to systemic or topical use of beta blockers were excluded. IOP was measured using the Goldmann applanation tonometer with the calibration scale masked from the examiner.

After informed consent patients were randomised to receive the two concentrations of drug in the order $A B A$ or BAB. 'A' corresponded to $0.5 \%$ and 'B' to $0.25 \%$ timolol, although the randomisation code was not broken until all patients had completed the study. Both eyes were treated simultaneously with the same concentration and entered into the study if the IOP in each was $30-49 \mathrm{mmHg}$. If one eye had an initial IOP of 21-29 mmHg it was also treated, but data points from it were not used in the study. The study period was 12 weeks for each patient, with changes in drug concentration at 4 and 8 weeks (Table I). IOPs were measured between $2 \mathrm{pm}$ and 4.30 pm every 2 weeks by one of the three investigators. This corresponds well with the period of relatively high IOP found in the diurnal cycle. ${ }^{5}$ Control of IOP over this period was felt to reflect accurately control over 24 hours. Patients whose IOP was 
Table I. Alternate treatment regimes for the two groups of patients

\begin{tabular}{lccc}
\hline & \multicolumn{3}{c}{ Strength (\%) of timolol } \\
\cline { 2 - 4 } Group & $0-4$ weeks & $4-8$ weeks & $8-12$ weeks \\
\hline ABA & 0.5 & 0.25 & 0.5 \\
BAB & 0.25 & 0.5 & 0.25 \\
\hline
\end{tabular}

Patients were randomised to receive the two concentrations of drug in the order $\mathrm{ABA}$ or $\mathrm{BAB}$. 'A' corresponded to $0.5 \%$ and ' $\mathrm{B}$ ' to $0.25 \%$ timolol, although the randomisation code was not broken until all patients had completed the study.

inadequately controlled were withdrawn from the study. Systemic or ocular side effects were recorded at each visit.

A placebo control group was not included because it was considered to be unethical to withhold treatment in patients with an initial IOP greater than $30 \mathrm{mmHg}$. For the same reason a washout period was not included at the changeover between concentrations of drug. Pressure measurements 2 weeks after the change of concentration were included in the protocol in an attempt to assess washout effects. Because this was a pharmacological study with a limited treatment period long-term disc and visual field changes were not monitored. The study design received local ethical committee approval.

To eliminate errors arising from inter-eye correlation, estimated at $r=0.9$ for IOP, ${ }^{6}$ data from pairs of eyes were averaged. As stated above, any eyes in which the initial IOP was below $30 \mathrm{mmHg}$ were excluded from this calculation.

Two sample (two-tailed) Student's $t$-tests were used to compare the initial fall in IOP between each strength. Paired $t$-tests were used to compare IOPs on changing strengths; $p$ values of less than 0.05 were considered statistically significant. Power analysis was carried out using nomograms. ${ }^{3,7}$

\section{RESULTS}

Forty patients were recruited over a period of 20 months between December 1989 and August 1991. Fifty-seven eyes had an initial IOP of $30-49 \mathrm{mmHg}$ and were therefore included in the study. Patients were randomised into the two treatment groups, receiving the trial drug in the order $\mathrm{ABA}$ or $\mathrm{BAB}$. Eyes were not randomised separately. Thirty-nine patients completed follow-up, and 1 patient was withdrawn after 4 weeks of treatment as a result of inadequate control of IOP in one eye. Initial IOP in this eye was $47 \mathrm{mmHg}$, the highest recorded IOP in the study; after 4 weeks it had fallen to $36 \mathrm{mmHg}$.

Table II. Initial fall in IOP

\begin{tabular}{lccc}
\hline & Timolol & Timolol & \\
& $0.25 \%$ & $0.5 \%$ & $p$ value \\
\hline$n$ & 20 & 20 & \\
Mean initial IOP $(\mathrm{mmHg})$ & 32.63 & 33.45 & $>0.1$ \\
Mean fall in IOP $(\mathrm{mmHg})$ & 11.31 & 12.03 & $>0.5$ \\
Standard deviation & 3.18 & 3.72 & \\
Mean \% fall in IOP & $34.67 \%$ & $35.95 \%$ & \\
\hline
\end{tabular}

The fall in IOP over the first 4 weeks of the study period for each patient. IOP readings after 2 and 4 weeks were combined. None of the differences reached statistical significance.
Table III. Effect of changing the concentration of timolol

\begin{tabular}{lcc}
\hline & Timolol & Timolol \\
& $0.25 \%$ to $0.5 \%$ & $0.5 \%$ to $0.25 \%$ \\
\hline$n$ & 39 & 39 \\
Mean change in IOP (mmHg) & -0.81 & +0.36 \\
Standard deviation & 2.59 & 3.17 \\
$p$ value & $>0.05$ & $>0.1$ \\
\hline
\end{tabular}

The effect of chnaging from one concentration of timolol to the other at the 4-week and 8-week clinic visits. There was no significant effect on IOP.

Both groups consisted of 11 men and 9 women. Group ABA had a mean age of 70.1 years and a mean initial IOP of $33.45 \mathrm{mmHg}$. The figures for group $\mathrm{BAB}$ were a mean age of 66.1 years and a mean initial IOP of $33.63 \mathrm{mmHg}$. There was no significant difference between the two groups in terms of age or initial IOPs.

The results of the study are summarised in Tables II-IV. There was no significant difference between the 2-week and 4-week IOP readings which were therefore combined. This was true both during the first 4 weeks of the study when there had been no previous treatment, and following changes in drug concentration at the 4-week and 8-week visits. Table II shows the initial fall in IOP during the first 4 weeks of the study. There was no statistically significant difference between the two groups. Table III shows the effect on IOP of changing from one concentration to another. Again there was no significant effect on IOP at the $p=0.05$ level. Table IV shows the mean levels of IOP maintained over the whole study period during treatment with the two different concentrations. Again these differences were not significant $(p>0.5)$.

Figure 1 is a scatter plot of the fall in IOP over the first 4 weeks of the study against mean initial IOP. It demonstrates that the percentage fall remained constant at approximately $35 \%$ for mean levels of IOP ranging from 30 to $40 \mathrm{mmHg}$. However, no statistical correlation was found after analysis of the data using Oldham's transformation. ${ }^{8}$ This approach was recommended by Gill et al. for studies of antihypertensive treatment, and is equally applicable to glaucoma therapy. ${ }^{9}$ Since the fall in IOP is the initial IOP (IOP1) minus the post-treatment IOP (IOP2), then IOP1 features in both variables if a direct comparison is made. This results inevitably in a positive correlation. Using Oldham's transformation, the fall in IOP was compared with the mean of the pre- and posttreatment values $(\mathrm{IOP} 1+\mathrm{IOP} 2) / 2$.

The two different concentrations of drug were both well tolerated. Fourteen patients complained of slight stinging

Table IV. Mean IOPs on treatment

\begin{tabular}{lcc}
\hline & Timolol & Timolol \\
& $0.25 \%$ & $0.5 \%$ \\
\hline Mean IOP (mmHg) & 21.12 & 20.75 \\
$n$ & 40 & 39 \\
Standard deviation & 3.63 & 3.18 \\
\hline
\end{tabular}

Mean IOPs of all patients on treatment during the whole study period. There was no significant difference between the two drugs $(p>0.5)$. 


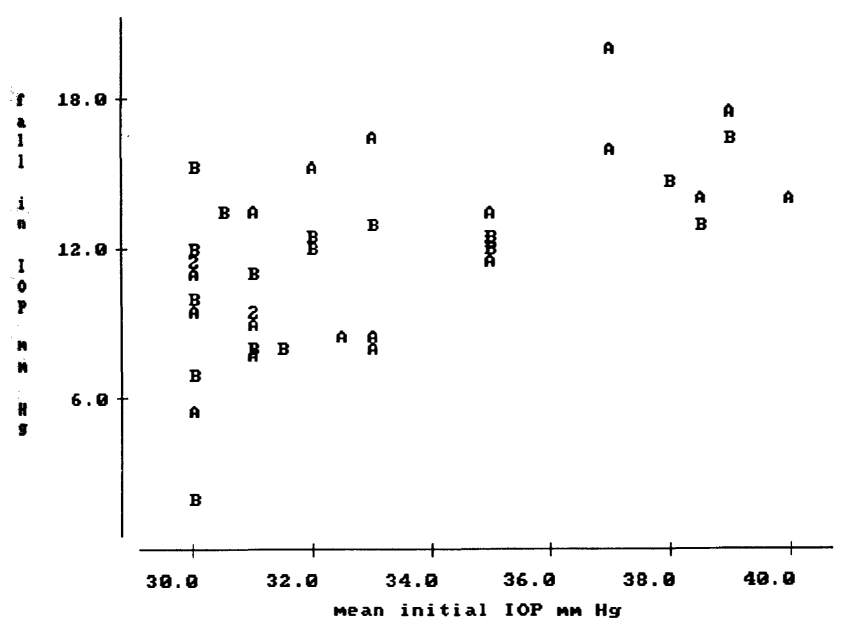

Fig. 1. Scatter diagram showing the fall in IOP over the initial 4-week study period, plotted against mean initial IOP, for the two concentrations of timolol. The 2-week and 4-week IOPs were combined. Eyes with initial IOPs below $30 \mathrm{mmHg}$ were excluded. A, $0.5 \%$ timolol; $B, 0.25 \%$ timolol; 2 , two data points at the same spot.

or burning following instillation of drops, at one or more points in the study. This occurred with both drops for 9 patients, with the $0.5 \%$ strength only in 3 patients and with the $0.25 \%$ strength only in 1 patient. Three patients complained of slight breathlessness, 1 with both drops and 1 each with the two different strengths.

Using nomograms ${ }^{3,7}$ we have calculated the power of this study to detect a difference in efficacy of $2 \mathrm{mmHg}$ to be in excess of $85 \%$.

\section{DISCUSSION}

In this study we found no evidence that timolol $0.5 \%$ is any more effective at lowering IOP than the $0.25 \%$ concentration. We also found no evidence that changing the strength of timolol produced any additional IOP lowering effect. These results are supported by the high statistical power of the study.

The two concentrations of timolol both produced an initial reduction of IOP of approximately 35\% (11.31 $\mathrm{mmHg}$ for $0.25 \%$ and $12.03 \mathrm{mmHg}$ for $0.5 \%$ ). These figures are higher than those obtained in most previous studies of timolol and other beta blockers. This reflects two factors. Firstly, the mean initial IOPs of patients in this study, $32.63 \mathrm{mmHg}(0.25 \%)$ and $33.45 \mathrm{mmHg}(0.5 \%)$, were high. None of the 56 studies quoted by Novack in his comprehensive review had higher initial IOPs. ${ }^{3}$ The mean reduction in initial IOP of 35\% was constant, hence the higher the initial IOP the greater the absolute fall in IOP (Fig. 1). Thus the large reduction of IOP that occurred in comparison with these other studies is not surprising.

Secondly, the initial fall was recorded over the first 4 weeks of the study period for each patient. This is before any effect of long-term drift would have developed. Mills reported that 8 of 30 patients initially controlled on timolol alone required supportive treatment to maintain normal IOPs, but only after 6-12 months follow-up.
One criticism of crossover studies is that an inadequate washout period may lead to erroneous results due to carryover effects from one drug to the next. In an effort to assess these effects, IOPs were recorded both at 2 and at 4 weeks after each change of drug concentration. Comparison of the 2-week and 4-week readings did not reveal any significant differences. This was true both for patients who changed from $0.5 \%$ to $0.25 \%$ timolol and vice versa. Of course this may be accounted for either by the fact that the two drugs were equally effective, or by an absence of carryover effects at and beyond 2 weeks. Further analysis of the data showed no significant differences in the levels of IOP maintained over the whole study period. These averaged approximately $21 \mathrm{mmHg}$, with standard deviations of $3.6 \mathrm{mmHg}(0.25 \%)$ and $3.18 \mathrm{mmHg}(0.5 \%)$ during treatment with both drug concentrations.

In 1983 Mills $^{1}$ concluded that there was 'little difference in effectiveness between long-term topical timolol $0.25 \%$ and timolol $0.5 \%$. Similarly, Uusitalo et al. ${ }^{2}$ found no difference in the IOP lowering effect of each strength in 1985. However, Novack ${ }^{3}$ reported that the probability of detecting a $2 \mathrm{mmHg}$ difference in efficacy between the two concentrations was only $21 \%$ and $28 \%$ respectively. This was due to the non-crossover design and relatively small numbers in each treatment group. In fact, Mills did find statistically significant differences at the 1-month and 12-month clinic visits. These differences occurred in both cases only for right eyes, and both favoured timolol $0.25 \%$. Additional evidence supporting our view that there is no therapeutic difference between the two strengths of timolol comes from studies of its concentration in the anterior chamber. These show that the level reached 1 hour after topical instillation is 1,000 times that required to block either beta- 1 or beta- 2 receptors. ${ }^{3}$ More recent work has shown that timolol concentrations as low as $0.008 \%$ have an ocular hypotensive effect. $^{10}$

Our study provides further evidence to support our belief that the substitution of timolol $0.25 \%$ for the 'stronger' $0.5 \%$, where IOP is inadequately controlled, is of no benefit. We suggest that the use of the $0.5 \%$ concentration could be abandoned since it has no therapeutic advantages and is more expensive. The relevance of our study is highlighted by annual sales figures from the manufacturers showing that sales of the $0.5 \%$ strength exceed those of the $0.25 \%$ concentration by 1.5 times $(1,826,700$ vs $1,179,900$ units $)$ at an additional cost of $£ 1.2$ million.

Key words: Raised intraocular pressure, Statistical power, Timolol $0.25 \%$ vs $0.5 \%$.

\section{REFERENCES}

1. Mills KB. Blind randomised non-crossover long-term trial comparing topical timolol $0.25 \%$ with timolol $0.5 \%$ in the treatment of simple chronic glaucoma. $\mathrm{Br} \mathrm{J}$ Ophthalmol 1983;67:216-9.

2. Uusitalo RJ, Palkama A, Stjernschantz J. A study of the efficacy of two commercial preparations of timolol maleate 
with special reference to side effects. Acta Ophthalmol (Copenh) 1985;63:634-41.

3. Novack GD. Ophthalmic beta-blockers since timolol. Surv Ophthalmol 1987;31:307-27.

4. Hickey-Dwyer M, Campbell SH, Harding SP. Double masked three period crossover investigation of metipranolol in control of raised intra-ocular pressure. J Ocular Pharmacol 1991;7:277-90.

5. Davson H. Physiology of the eye. London: Macmillan, 1990:50-1.

6. Ray WA, O'Day DM. Statistical analysis of multi-eye data in ophthalmic research. Invest Ophthalmol Vis Sci 1985;26: 1186-8.
7. Gore SM, Altman DG. Statistics in practice. London: British Medical Association, 1982:6-8.

8. Oldham PD. Measurement in medicine: the interpretation of numerical data. London: English Universities Press, 1968: 148-52.

9. Gill JS, Zezulka AV, Beevers DG, Davies P. Relation between initial blood pressure and its fall with treatment. Lancet 1985; 1:567-9.

10. Mottow-Lippa LS, Lippa EA, Naidoff MA, Clementi R, Bjornsson T, Jones K. 0.008\% timolol ophthalmic solution: a minimal-effect dose in a normal volunteer model. Arch Ophthalmol 1990;108:61-4. 